\title{
Index mots clés
}

\section{Keywords index}

(C) Lavoisier SAS 2018

A

Amputation - Appareillage des membres inférieurs chez l'amputé diabétique : prothèse et chaussures, 35

Arthrodèse - Arthrodèse tibiotalocalcanéenne par enclouage transplantaire rétrograde en milieu septique : note technique ; à propos de six cas, 30

Arthrose sous-talienne-Facteurs d'irréductibilité des luxations sous-taliennes latérales. Étude de trois cas et revue de la littérature, 15

Articulation sous-talienne - Facteurs d'irréductibilité des luxations sous-taliennes latérales. Étude de trois cas et revue de la littérature, 15

\section{B}

Botryomycose-Lésion granulomateuse exceptionnelle chez un homme immunocompétent, 53

Brièveté - Brièveté des gastrocnémiens et pathologies du pied. Une physiopathologie controversée, 49

\section{C}

Capsulorraphie médiale - Ligamentoplastie médiale dans le traitement de l'hallux valgus, 2

Carcinome épidermoïde - Lésion granulomateuse exceptionnelle chez un homme immunocompétent, 53

Chaussure-Appareillage des membres inférieurs chez l'amputé diabétique : prothèse et chaussures, 35

Cheville - Arthrodèse tibiotalocalcanéenne par enclouage transplantaire rétrograde en milieu septique: note technique ; à propos de six cas, 30

Chondrocyte - Le ménisque de la première articulation métatarsophalangienne peut-il expliquer la douleur en regard du bunion chez les sujets jeunes? Étude anatomopathologique à propos de 30 hallux valgus opérés, 70

\section{D}

Diabète-Appareillage des membres inférieurs chez l'amputé diabétique : prothèse et chaussures, 35

DMAA - Le ménisque de la première articulation métatarsophalangienne peut-il expliquer la douleur en regard du bunion chez les sujets jeunes? Étude anatomopathologique à propos de 30 hallux valgus opérés, 70

Douleur de la cheville - Tumeur glomique extradigitale : une cause rare de douleur chronique de cheville, 27

Duplication d'orteil - Une hexadactylie douloureuse, 76

\section{E}

Élastographie par ondes de cisaillement - Bases physiques de l'élastographie et applications au tendon calcanéen normal et pathologique, 7

Élastographie quasi-statique - Bases physiques de l'élastographie et applications au tendon calcanéen normal et pathologique, 7

Élastosonographie - Bases physiques de l'élastographie et applications au tendon calcanéen normal et pathologique, 7

Embrochage - Un cas inhabituel de luxation tibiotalaire postéromédiale pure ouverte suite à un accident de ski, 56

Enclouage - Arthrodèse tibiotalocalcanéenne par enclouage transplantaire rétrograde en milieu septique : note technique ; à propos de six cas, 30

\section{G}

Gastrocnémien - Brièveté des gastrocnémiens et pathologies du pied. Une physiopathologie controversée, 49

\section{H}

Hallux valgus - Le ménisque de la première articulation métatarsophalangienne peut-il expliquer la douleur en 
regard du bunion chez les sujets jeunes ? Étude anatomopathologique à propos de 30 hallux valgus opérés, 70

Hallux valgus - Ligamentoplastie médiale dans le traitement de l'hallux valgus, 2

$\mathbf{L}$

Lésions associées- Facteurs d'irréductibilité des luxations sous-taliennes latérales. Étude de trois cas et revue de la littérature, 15

Luxation - Facteurs d'irréductibilité des luxations soustaliennes latérales. Étude de trois cas et revue de la littérature, 15

Luxation tibiotalienne - Un cas inhabituel de luxation tibiotalaire postéromédiale pure ouverte suite à un accident de ski, 56

\section{$\mathbf{M}$}

Marche-Brièveté des gastrocnémiens et pathologies du pied. Une physiopathologie controversée, 49

Ménisque - Le ménisque de la première articulation métatarsophalangienne peut-il expliquer la douleur en regard du bunion chez les sujets jeunes? Étude anatomopathologique à propos de 30 hallux valgus opérés, 70

Métatarsalgie - Une hexadactylie douloureuse, 76

Mycoses - Lésion granulomateuse exceptionnelle chez un homme immunocompétent, 53

\section{$\mathbf{N}$}

Nerf - Le ménisque de la première articulation métatarsophalangienne peut-il expliquer la douleur en regard du bunion chez les sujets jeunes? Étude anatomopathologique à propos de 30 hallux valgus opérés, 70

\section{$\mathbf{O}$}

Orteil surnuméraire- Une hexadactylie douloureuse, 76
Orthèse plantaire - Aide à la prescription d'orthèses plantaires, 82

\section{$\mathbf{P}$}

Phénomène de Splendore-Hoeppli-Lésion granulomateuse exceptionnelle chez un homme immunocompétent, 53

Pied - Appareillage des membres inférieurs chez l'amputé diabétique : prothèse et chaussures, 35

Polydactylie - Une hexadactylie douloureuse, 76

Prescription médicale - Aide à la prescription d'orthèses plantaires, 82

Prothèse-Appareillage des membres inférieurs chez l'amputé diabétique : prothèse et chaussures, 35

\section{S}

Staphylococcus aureus-Lésion granulomateuse exceptionnelle chez un homme immunocompétent, 53

Syndactylie- Une hexadactylie douloureuse, 76

Syndesmose - Un cas inhabituel de luxation tibiotalaire postéromédiale pure ouverte suite à un accident de ski, 56

\section{$\mathbf{T}$}

Tendinopathie - Bases physiques de l'élastographie et applications au tendon calcanéen normal et pathologique, 7

Tendon calcanéen - Bases physiques de l'élastographie et applications au tendon calcanéen normal et pathologique, 7

Tendon d'Achille - Bases physiques de l'élastographie et applications au tendon calcanéen normal et pathologique, 7

Tumeur glomique - Tumeur glomique extradigitale : une cause rare de douleur chronique de cheville, 27 